\title{
Mean Time to Recruitment for A Multi Grade Manpower System with Single Threshold, Single Source of Depletion When Inter Policy Decisions Form an Order Statistics.
}

\author{
K.Srividhya ${ }^{1}$, S.Sendhamizhselvi ${ }^{2}$ \\ I'Assistant professor, Department of Mathematics, National College, Trichy, Tamil Nadu, India-620001), \\ ${ }_{2}^{2}$ (Assistant professor, PG and Research Department of Mathematics, Government Arts College,Trichy-620022, \\ Tamil Nadu, India.)
}

\begin{abstract}
In this paper, for a marketing organization consisting of multi grades subject to the depletion of manpower (wastages) due to policy decisions with high or low attrition rate, is considered. An important system characteristic namely the mean time to recruitment is obtained for a suitable policy of recruitment when (i) wastages are independent and identically distributed exponential random variable and (ii) threshold for each grade has single component with exponential distribution and (iii) the inter-policy decisions form an order statistics.
\end{abstract}

Keywords: Hyper exponential, Loss of manpower(wastage),Order Statistics, Mean time to recruitment, Threshold with single components, Two types of policy decisions with high or low attrition rate,

\section{Introduction}

Exits of personal which is in other words known as wastage, is an important aspects in the study of manpower planning. Many models have been discussed using different types of wastages and also different types of distribution for the loss of man powers, the thresholds and inter decision times. Such models are seen in [6] and [7].In [1],[2],[3] and [4] the authors have obtained the mean time to recruitment in a two grade manpower system based on order statistics by assuming different distribution for thresholds.

The problem of time to recruitment is studied by several authors for the organizations consisting of single grade/two grade/ three grades. More specifically for a two grade system, In all the earlier work, the threshold for the organization is minimum or maximum or sum of the thresholds for the loss of manpower in each grades, no attempt has been made so far to design a comprehensive recruitment policy for a system with two or three grades . In [8] a new design for a comprehensive univariate CUM recruitment policy of manpower system is used with ' $\mathrm{n}$ ' grades in order to bring results proved independently for maximum, minimum model as a special case.

In all previous work, the problem of time to recruitment is studied for only an organization consisting of at most three grades In this paper an organization with $\mathrm{n}$-grades is considered and the mean time to recruitment are obtained using an appropriate univariate CUM policy of recruitment (i.e) " The organization survives iff atleast $r,(1 \leq r \leq n)$ out of $n$-grades survives in the sense that threshold crossing has not take place in these grades." when the inter decision time form order statistics.

\section{Model Description And Analysis}

An organization with n-grades which takes policy decisions at random epoch is considered. At every decision making epoch, a random number of persons quit the organization. There is an associated loss of man hours to the organization if the person quits. The loss of manhours forms a sequence of independent and identically distributed random variables. Each grade has its own thresholds. The inter decision time forms an order statistics. The loss of manpower process, inter decision time process and the thresholds are statistically independent. The thresholds for the $\mathrm{n}$ - grades are independently and identically distributed exponential random variables.

Let $X_{i}, i=1,2,3 \ldots$ is a continuous random variable denoting the amount of depletion caused to the system due to the exit of persons corresponding to the $\mathrm{i}^{\text {th }}$ decision, $\mathrm{t}_{\mathrm{i}}$, time of occurrence of the $\mathrm{i}^{\text {th }}$ decision. $\mathrm{G}_{\mathrm{k}}($.$) :$ Cumulative distribution function of $\sum_{\mathrm{i}=1}^{\mathrm{n}} \mathrm{x}_{\mathrm{i}}$. Let $\mathrm{U}_{\mathrm{i}}$, Independent and identically distributed random variables denoting the time between $(\mathrm{i}-1)^{\text {th }}$ and $\mathrm{i}^{\text {th }}$ decision making epoch, $\mathrm{i}=1,2, \ldots \mathrm{f}($.$) the Probability density function$ of $U_{i}, i=1,2, \ldots f_{k}()$.$k -fold convolution of f(),. \bar{f}($.$) : Laplace transform of f($.$) . Let V_{k}(t)$ be the Probability that there are exactly $\mathrm{k}$ decision making epochs in $(0, \mathrm{t}] . \mathrm{Y}_{\mathrm{j}}$ a continuous random variable denoting the threshold level for the $\mathrm{j}^{\text {th }}$ grade. $\mathrm{Y}$ be a continuous random variable denoting the threshold level for the organization. $\mathrm{H}($.$) the distribution$ function of $Y$. Let $T_{j}$ be the time taken for threshold crossing in the $j^{\text {th }}$ grade, $j=1,2, \ldots n$. T time for recruitment in the organization. $\mathrm{E}(\mathrm{T})$ : Mean time for recruitment. 


\section{MAIN RESULTS}

The survival function of the time to recruitment is given by

$$
\begin{aligned}
& P(T>t)=\sum_{k=0}^{\infty} P(\text { Exactly } k \text { decision epoch }(0, t] \text { and the threshold level } Y \text { is not crossed } \\
& \text { by the total loss of manhours in these } k \text { decisions in atleast } r \text { grades }) \\
& \text { i. e } P(T>t)=\sum_{k=0}^{\infty} V_{k}(t) P\left(\sum_{i=1}^{k} X_{i}<Y\right)
\end{aligned}
$$

By the law of total probability

$$
\begin{gathered}
P\left(\sum_{i=1}^{k} X_{i}<Y\right)=\int_{0}^{\infty} P\left[Y>\sum_{i=1}^{k} X_{i} / \sum_{i=1}^{k} X_{i}=x\right] g_{k}(x) d x \\
=\int_{0}^{\infty} g_{k}(x)[1-H(x)] d x . \\
=\int_{0}^{\infty} g_{k}(x) \sum_{i=r}^{n} n C_{i}[1-H(x)]^{i}[H(x)]^{n-i} d x . \\
=\int_{0}^{\infty} g_{k}(x) \sum_{i=r}^{n} n C_{i}\left[e^{-\theta x}\right]^{i}\left[1-e^{-\theta x}\right]^{n-i} d x . \\
=\sum_{i=r}^{n} n_{i} \int_{0}^{\infty} g_{k}(x) e^{-i \theta x}\left[1-e^{-\theta x}\right]^{n-i} d x
\end{gathered}
$$

Using binomial expansion

$$
\begin{array}{r}
=\sum_{i=r}^{n} n_{i} \int_{0}^{\infty} g_{k}(x) e^{-i \theta x}\left[1-(n-i) C_{1} e^{-\theta x}+(n-i) C_{2} e^{-2 \theta x}+\ldots(-1)^{n-i} e^{-(n-i) \theta x}\right] d x \\
=\sum_{i=r}^{n} n_{i} \int_{0}^{\infty} g_{k}(x)\left[e^{-i \theta x}-(n-i) C_{1} e^{-(i+1) \theta x}+(n-i) C_{2} e^{-(i+2) \theta x}+\right. \\
\left.=\sum_{i=r}^{n} n_{i}\left[[\bar{g}(i \theta)]^{k}-(n-i) C_{1}[\bar{g}((i+1) \theta)]^{k}+(n-i) C_{2}[\bar{g}((i+2) \theta)]^{k}\right]+\ldots(-1)^{n-i} e^{n-i n \theta x}\right] d x
\end{array}
$$

From renewal theory $V_{k}(t)=F_{k}(t)-F_{k+1}(t)$ with $F_{0}(t)=1$

Substituting (3) and (4) in (1) we get,

$$
\begin{gathered}
\mathrm{P}(\mathrm{T}>\mathrm{t})=\sum_{\mathrm{k}=0}^{\infty}\left[\mathrm{F}_{\mathrm{k}}(\mathrm{t})-\mathrm{F}_{\mathrm{k}+1}(\mathrm{t})\right] \sum_{\mathrm{i}=\mathrm{r}}^{\mathrm{n}} \mathrm{nC}_{\mathrm{i}}\left[\left[\overline{\mathrm{g}}(\mathrm{i} \theta]^{\mathrm{k}}-(\mathrm{n}-\mathrm{i}) \mathrm{C}_{1}[\overline{\mathrm{g}}((\mathrm{i}+1) \theta)]^{\mathrm{k}}+(\mathrm{n}-\mathrm{i}) \mathrm{C}_{2}[\overline{\mathrm{g}}((\mathrm{i}+2) \theta)]^{\mathrm{k}}\right]\right. \\
\left.+\ldots+(-1)^{\mathrm{n}-\mathrm{i}}[\overline{\mathrm{g}}(\mathrm{n} \theta)]^{\mathrm{k}}\right]
\end{gathered}
$$

Expanding and simplifying we get

$$
\begin{aligned}
& P(T>t) \\
& =\sum_{i=r}^{n} \mathrm{nC}_{\mathrm{i}}\left\{\begin{array}{c}
\left.1[-[1-\overline{\mathrm{g}}(\mathrm{i} \theta)]] \sum_{\mathrm{k}=1}^{\infty} \mathrm{F}_{\mathrm{k}}(\mathrm{t})[\overline{\mathrm{g}}(\mathrm{i} \theta)]^{\mathrm{k}-1}-(\mathrm{n}-\mathrm{i}) \mathrm{C}_{1}[1-[1-\overline{\mathrm{g}}((\mathrm{i}+1) \theta))]\right] \sum_{\mathrm{k}=1}^{\infty} \mathrm{F}_{\mathrm{k}}(\mathrm{t})[\overline{\mathrm{g}}((\mathrm{i}+1) \theta)]^{\mathrm{k}-1} \\
+\cdots(-1)^{\mathrm{n}-\mathrm{i}}[1-[1-\overline{\mathrm{g}}(\mathrm{n} \theta)]] \sum_{\mathrm{k}=1}^{\infty} \mathrm{F}_{\mathrm{k}}(\mathrm{t})[\overline{\mathrm{g}}((\mathrm{n} \theta))]^{\mathrm{k}-1}
\end{array}\right.
\end{aligned}
$$




$$
\begin{aligned}
\mathrm{P}(\mathrm{T}>\mathrm{t})=-\sum_{\mathrm{i}=\mathrm{r}}^{\mathrm{n}} \mathrm{nC}_{\mathrm{i}}\left\{[1-\overline{\mathrm{g}}(\mathrm{i} \theta)] \sum_{\mathrm{k}=1}^{\infty} \mathrm{F}_{\mathrm{k}}(\mathrm{t})[\overline{\mathrm{g}}(\mathrm{i} \theta)]^{\mathrm{k}-1}\right. \\
\quad-(\mathrm{n}-\mathrm{i}) \mathrm{C}_{1}[1-\overline{\mathrm{g}}((\mathrm{i}+1) \theta)] \sum_{\mathrm{k}=1}^{\infty} \mathrm{F}_{\mathrm{k}}(\mathrm{t})[\overline{\mathrm{g}}((\mathrm{i}+1) \theta)]^{\mathrm{k}-1} \\
\left.+\cdots(-1)^{\mathrm{n}-\mathrm{i}}[1-\overline{\mathrm{g}}(\mathrm{n} \theta)] \sum_{\mathrm{k}=1}^{\infty} \mathrm{F}_{\mathrm{k}}(\mathrm{t})[\overline{\mathrm{g}}((\mathrm{n} \theta))]^{\mathrm{k}-1}\right\}
\end{aligned}
$$

From equation (6), the distribution function of the time for recruitment is given by

$$
\begin{aligned}
& \mathrm{L}(\mathrm{t})=1-\mathrm{P}(\mathrm{T}>\mathrm{t}) \\
& \begin{aligned}
=1+\sum_{\mathrm{i}=\mathrm{r}}^{\mathrm{n}} \mathrm{nC}_{\mathrm{i}}\left\{[ 1 - \overline { \mathrm { g } } ( \mathrm { i } \theta ) ] \sum _ { \mathrm { k } = 1 } ^ { \infty } \mathrm { F } _ { \mathrm { k } } ( \mathrm { t } ) \left[\overline{\mathrm{g}}(\mathrm{i} \theta]^{\mathrm{k}-1}\right.\right. \\
\left.\quad-(\mathrm{n}-\mathrm{i}) \mathrm{C}_{1}[1-\overline{\mathrm{g}}((\mathrm{i}+1) \theta))\right] \sum_{\mathrm{k}=1}^{\infty} \mathrm{F}_{\mathrm{k}}(\mathrm{t})[\overline{\mathrm{g}}((\mathrm{i}+1) \theta)]^{\mathrm{k}-1} \\
\left.+\cdots(-1)^{n-i} \quad[1-\overline{\mathrm{g}}(\mathrm{n} \theta)] \sum_{\mathrm{k}=1}^{\infty} \mathrm{F}_{\mathrm{k}}(\mathrm{t})[\overline{\mathrm{g}}((\mathrm{n} \theta))]^{\mathrm{k}-1}\right\}
\end{aligned}
\end{aligned}
$$

Differentiating with respect to $t$

$l(t)=$

$$
\begin{aligned}
& \sum_{\mathrm{i}=\mathrm{r}}^{\mathrm{n}} \mathrm{nC}_{\mathrm{i}}\left\{[ 1 - \overline { \mathrm { g } } ( \mathrm { i } \theta ) ] \sum _ { \mathrm { k } = 1 } ^ { \infty } \mathrm { f } _ { \mathrm { k } } ( \mathrm { t } ) \left[\overline{\mathrm{g}}(\mathrm{i} \theta]^{\mathrm{k}-1}\right.\right. \\
&\left.-(\mathrm{n}-\mathrm{i}) \mathrm{C}_{1}[1-\overline{\mathrm{g}}((\mathrm{i}+1) \theta))\right] \sum_{\mathrm{k}=1}^{\infty} \mathrm{f}_{\mathrm{k}}(\mathrm{t})[\overline{\mathrm{g}}((\mathrm{i}+1) \theta)]^{\mathrm{k}-1} \\
&\left.+\cdots \quad(-1)^{n-i} \quad[1-\overline{\mathrm{g}}(\mathrm{n} \theta)] \sum_{\mathrm{k}=1}^{\infty} \mathrm{f}_{\mathrm{k}}(\mathrm{t})[\overline{\mathrm{g}}((\mathrm{n} \theta))]^{\mathrm{k}-1}\right\}
\end{aligned}
$$

Taking Laplace transform on both sides,

$$
\begin{aligned}
& \overline{\mathrm{l}}(\mathrm{s})=\sum_{\mathrm{i}=\mathrm{r}}^{\mathrm{n}} \mathrm{nC} \mathrm{C}_{\mathrm{i}}\left\{[ 1 - \overline { \mathrm { g } } ( \mathrm { i } \theta ) ] \sum _ { \mathrm { k } = 1 } ^ { \infty } [ \overline { \mathrm { f } } ( \mathrm { s } ) ] ^ { \mathrm { k } } \left[\overline{\mathrm{g}}(\mathrm{i} \theta]^{\mathrm{k}-1}\right.\right. \\
& -(n-i) C_{1}[1-\bar{g}((i+1) \theta)] \sum_{k=1}^{\infty}[\bar{f}(s)]^{k}\left[\bar{g}((i+1) \theta]^{k-1}+\right. \\
& (-1)^{\mathrm{n}-\mathrm{i}}[1-\overline{\mathrm{g}}(\mathrm{n} \theta)] \sum_{\mathrm{k}=1}^{\infty}[\overline{\mathrm{f}}(\mathrm{s})]^{\mathrm{k}}[\overline{\mathrm{g}}((\mathrm{n} \theta))]^{\mathrm{k}-1} \\
& \bar{l}(s)=\sum_{i=r}^{n} n C_{i}\left\{[ 1 - \overline { g } ( i \theta ) ] \overline { f } ( s ) \sum _ { k = 1 } ^ { \infty } [ \overline { f } ( s ) ] ^ { k - 1 } \left[\bar{g}(i \theta]^{k-1}\right.\right. \\
& -(n-i) C_{1}[1-\bar{g}((i+1) \theta)] \bar{f}(s) \sum_{k=1}^{\infty}[\bar{f}(s)]^{k-1}\left[\bar{g}((i+1) \theta]^{k-1}+\right. \\
& \ldots(-1)^{n-i}[1-\bar{g}(n \theta)] \bar{f}(s) \sum_{k=1}[\bar{f}(s)]^{k-1}[\bar{g}((n \theta))]^{k-1} \\
& \bar{l}(s)=\sum_{i=r}^{n} n C_{i}\left\{\frac{[1-\bar{g}(i \theta)] \bar{f}(s)}{[1-\bar{g}(i \theta) \bar{f}(s)]}-(n-i) C_{1} \frac{[1-\bar{g}((i+1) \theta)] \bar{f}(s)}{[1-\bar{g}((i+1) \theta) \bar{f}(s)]}+\cdots(-1)^{n-i} \frac{[1-\bar{g}(n \theta)] \bar{f}(s)}{[1-\bar{g}(n \theta) \bar{f}(s)]}\right\} \\
& E(T)=-\left[\frac{d}{d s}(\bar{l}(s)]_{s=0}\right.
\end{aligned}
$$




$$
\begin{aligned}
=-\sum_{i=r}^{n} n C_{i} & \left\{\frac{[1-\bar{g}(i \theta)]\left[\frac{d}{d s}(\bar{f}(s)]_{s=0}\right.}{[1-\bar{g}(i \theta) \bar{f}(s)]_{s=0}^{2}}-(n-i) C_{1} \frac{[1-\bar{g}((i+1) \theta)]\left[\frac{d}{d s}(\bar{f}(s)]_{s=0}\right.}{[1-\bar{g}((i+1) \theta) \bar{f}(s)]_{s=0}^{2}}+\cdots\right. \\
\left.+(-1)^{n-i} \frac{[1-\bar{g}(n \theta)]\left[\frac{d}{d s}(\bar{f}(s)]_{s=0}\right.}{[1-\bar{g}(i \theta) \bar{f}(s)]_{s=0}^{2}}\right\} & (11)
\end{aligned}
$$

Since probability density function of loss of manpower $\mathrm{g}(\mathrm{x})$ is an exponential with parameter $\mu$

$$
\bar{g}(s)=\frac{\mu}{\mu+s}
$$

Consider the population $\left\{U_{i}\right\}_{i=1}^{\infty}$ of independent and identically distributed interdecision times with hyper exponential cumulative distribution $F(t)=1-p e^{-\lambda_{\mathrm{h}} t}-q e^{-\lambda_{l} t}$ and the corresponding density function $\mathrm{f}(\mathrm{t})$.Assume that $\left\{U_{i}\right\}_{i=1}^{m}$ be a sample of size $\mathrm{m}$ selected from this population .Let $U_{1}, U_{2}, \ldots, U_{m}$ be the order statistics corresponding to this sample with respective probability density function $f_{U_{1}}, f_{U_{2}}, \ldots . . f_{U_{m}} . U_{1}$ is the first order statistics and $U_{m}$ is the $\mathrm{m}^{\text {th }}$ order statistics such that $U_{1} \leq U_{2} \leq \ldots \ldots \leq U_{m}$ and hence not independent. The probability density function of $\mathrm{j}^{\text {th }}$ order statistics is given by [Sheldon M.Ross2005]

$$
f_{U_{j}}(t)=j\left(\begin{array}{c}
m \\
j
\end{array}\right)[F(t)]^{j-i} f(t)[1-F(t)]^{m-j}, j=1,2,3 \ldots m
$$

Therefore the probability density function of $U_{1}$ and $U_{m}$ are given by

Case(i):

$$
\begin{gathered}
f_{U_{1}}(t)=m f(t)[1-F(t)]^{m-1} \\
f_{U_{m}}(t)=m f(t)[F(t)]^{m-1}
\end{gathered}
$$

$$
\begin{aligned}
& f(t)=f_{U_{1}}(t) \\
& \bar{f}(s)=\bar{f}_{U_{1}}(s)=\int_{0}^{\infty} e^{-s t} m f(t)[1-F(t)]^{m-1} \\
&= \int_{0}^{\infty} e^{-s t}\left[-[d(1-F(t))]^{m}\right] \\
& \bar{f}(s)=\int_{0}^{\infty} e^{-s t}\left[-\left(d\left(p e^{-\lambda_{\mathrm{h}} t}+q e^{-\lambda_{l} t}\right)^{m}\right]\right.
\end{aligned}
$$

By using binomial expansion

$$
\begin{aligned}
& \bar{f}(s)=\int_{0}^{\infty} e^{-s t}\left[-d\left(\sum_{r_{1}=0}^{m}\left(\begin{array}{l}
m \\
r_{1}
\end{array}\right) p^{r_{1}} q^{m-r_{1}} e^{-\left(\lambda_{\mathrm{h}} r_{1}-\lambda_{l} r_{1}+\lambda_{l} m\right) t}\right)\right] \\
& =\sum_{r_{1}=0}^{m}\left(\begin{array}{l}
m \\
r_{1}
\end{array}\right) p^{r_{1}} q^{m-r_{1}} \int_{0}^{\infty} e^{-s t}\left(\lambda_{\mathrm{h}} r_{1}-\lambda_{l} r_{1}+\lambda_{l} m\right) e^{-\left(\lambda_{\mathrm{h}} r_{1}-\lambda_{l} r_{1}+\lambda_{l} m\right) t} d t \\
& =\sum_{r_{1}=0}^{m}\left(\begin{array}{l}
m \\
r_{1}
\end{array}\right) p^{r_{1}} q^{m-r_{1}}\left(\lambda_{\mathrm{h}} r_{1}-\lambda_{l} r_{1}+\lambda_{l} m\right) \int_{0}^{\infty} e^{-\left(s+\lambda_{\mathrm{h}} r_{1}-\lambda_{l} r_{1}+\lambda_{l} m\right) t} d t \\
& =\sum_{r_{1}=0}^{m}\left(\begin{array}{l}
m \\
r_{1}
\end{array}\right) p^{r_{1}} q^{m-r_{1}}\left(\lambda_{\mathrm{h}} r_{1}-\lambda_{l} r_{1}+\lambda_{l} m\right)\left[\frac{e^{-\left(s+\lambda_{\mathrm{h}} r_{1}-\lambda_{l} r_{1}+\lambda_{l} m\right) t}}{-\left(s+\lambda_{\mathrm{h}} r_{1}-\lambda_{l} r_{1}+\lambda_{l} m\right) t}\right]_{0}^{\infty} \\
& =\sum_{r_{1}=0}^{m}\left(\begin{array}{l}
m \\
r_{1}
\end{array}\right) p^{r_{1}} q^{m-r_{1}}\left(\lambda_{\mathrm{h}} r_{1}-\lambda_{l} r_{1}+\lambda_{l} m\right)\left[\frac{1}{\left(s+\lambda_{\mathrm{h}} r_{1}-\lambda_{l} r_{1}+\lambda_{l} m\right)}\right] \\
& \frac{d}{d s}(\bar{f}(s))=\sum_{r_{1}=0}^{m}\left(\begin{array}{l}
m \\
r_{1}
\end{array}\right) p^{r_{1}} q^{m-r_{1}}\left(\lambda_{\mathrm{h}} r_{1}-\lambda_{l} r_{1}+\lambda_{l} m\right)\left[\frac{-1}{\left(s+\lambda_{\mathrm{h}} r_{1}-\lambda_{l} r_{1}+\lambda_{l} m\right)^{2}}\right] \\
& {\left[\frac{d}{d s}(\bar{f}(s))\right]_{s=0}=-\sum_{r_{1}=0}^{m}\left(\begin{array}{l}
m \\
r_{1}
\end{array}\right) p^{r_{1}} q^{m-r_{1}}\left[\frac{1}{\left(\lambda_{\mathrm{h}} r_{1}-\lambda_{l} r_{1}+\lambda_{l} m\right)}\right]} \\
& \&(\bar{f}(s))_{s=0}=1
\end{aligned}
$$


Substituting the equation (12) \& (14) in (11)

$$
\begin{aligned}
& E(T)=-\sum_{i=r}^{n} n C_{i}\left\{\frac{\left[\frac{d}{d s}(\bar{f}(s)]_{s=0}\right.}{[1-\bar{g}(i \theta)]}-(n-i) C_{11} \frac{\left[\frac{d}{d s}(\bar{f}(s)]_{s=0}\right.}{[1-\bar{g}((i+1) \theta)]}+\cdots+(-1)^{n-i} \frac{\left[\frac{d}{d s}(\bar{f}(s)]_{s=0}\right.}{[1-\bar{g}(n \theta)]}\right. \\
& E(T)=-\sum_{i=r}^{n} n C_{i}\left\{\frac{1}{[1-\bar{g}(i \theta)]}-(n-i) C_{1} \frac{1}{[1-\bar{g}((i+1) \theta)]}+\cdots+(-1)^{n-i} \frac{1}{[1-\bar{g}(n \theta)]}\right\} \cdot\left[\frac{d}{d s}(\bar{f}(s)]_{s=0}\right. \\
& E(T)=-\sum_{i=r}^{n} n C_{i}\left\{\frac{\mu+i \theta}{i \theta}-(n-i) C_{1} \frac{\mu+(i+1) \theta}{(i+1) \theta}+\cdots+(-1)^{n-i} \frac{\mu+n \theta}{n \theta}\right\} \cdot\left[\frac{d}{d s}(\bar{f}(s)]_{s=0}\right.
\end{aligned}
$$

Substituting $\left[\frac{d}{d s}(\bar{f}(s)]_{s=0}\right.$ from (13) in (15), we obtain the mean time to recruitment,

$$
\begin{array}{r}
E(T)=\sum_{i=r}^{n} n C_{i}\left\{\frac{\mu+i \theta}{i \theta}-(n-i) C_{1} \frac{\mu+(i+1) \theta}{(i+1) \theta}+\cdots+(-1)^{n-i} \frac{\mu+n \theta}{n \theta}\right\} \times \\
\sum_{r_{1}=0}^{m}\left(\begin{array}{l}
m \\
r_{1}
\end{array}\right) p^{r_{1}} q^{m-r_{1}}\left[\frac{1}{\left(\lambda_{\mathrm{h}} r_{1}-\lambda_{l} r_{1}+\lambda_{l} m\right)}\right]
\end{array}
$$

Case(ii):

$$
\begin{aligned}
& f(t)=f_{U_{m}}(t)=m f(t)[F(t)]^{m-1} \\
& \bar{f}(s)=\bar{f}_{U_{m}}(s)=\int_{0}^{\infty} e^{-s t} m f(t)[F(t)]^{m-1} d t \\
& =\int_{0}^{\infty} e^{-s t} d(F(t))^{m}=\int_{0}^{\infty} e^{-s t}\left[d\left(1-p e^{-\lambda_{\mathrm{h}} t}-q e^{-\lambda_{l} t}\right)\right]^{m} \\
& =\int_{0}^{\infty} e^{-s t} d\left(\sum_{r_{1}=0}^{m}\left(\begin{array}{l}
m \\
r_{1}
\end{array}\right)(-1)^{m-r_{1}} 1^{r_{1}}\left(p e^{-\lambda_{\mathrm{h}} t}+q e^{-\lambda_{l} t}\right)^{m-r_{1}}\right. \\
& =\int_{0}^{\infty} e^{-s t} d\left(\sum_{r_{1}=0}^{m} \sum_{r_{2}=0}^{m-r_{1}}(-1)^{m-r_{1}} \frac{m !}{r_{1} ! r_{2} !\left(m-r_{1}-r_{2}\right) !} p^{r_{2}} q^{m-r_{1}-r_{2}} e^{-\left(\lambda_{\mathrm{h}} r_{2}-\lambda_{l} r_{2}-\lambda_{l} r_{1}+\lambda_{l} m\right) t}\right) \\
& =\int_{0}^{\infty} e^{-s t} \sum_{r_{1}=0}^{m} \sum_{r_{2}=0}^{m-r_{1}} \frac{(-1)^{m-r_{1}} m ! p^{r_{2}} q^{m-r_{1}-r_{2}}\left(\lambda_{\mathrm{h}} r_{2}-\lambda_{l} r_{2}-\lambda_{l} r_{1}+\lambda_{l} m\right)}{r_{1} ! r_{2} !\left(m-r_{1}-r_{2}\right) !} e^{-\left(\lambda_{\mathrm{h}} r_{2}-\lambda_{l} r_{2}-\lambda_{l} r_{1}+\lambda_{l} m\right) t} d t \\
& =\sum_{r_{1}=0}^{m} \sum_{r_{2}=0}^{m-r_{1}}(-1)^{m-r_{1}+1} \frac{m !}{r_{1} ! r_{2} !\left(m-r_{1}-r_{2}\right) !} p^{r_{2}} q^{m-r_{1}-r_{2}}\left(\lambda_{\mathrm{h}} r_{2}-\lambda_{l} r_{2}-\lambda_{l} r_{1}+\lambda_{l} m\right) \\
& \times \int_{0}^{\infty} e^{-\left(s+\lambda_{\mathrm{h}} r_{2}-\lambda_{l} r_{2}-\lambda_{l} r_{1}+\lambda_{l} m\right) t} d t \\
& \bar{f}(s)=\sum_{r_{1}=0}^{m} \sum_{r_{2}=0}^{m-r_{1}}(-1)^{m-r_{1}+1} \frac{m !}{r_{1} ! r_{2} !\left(m-r_{1}-r_{2}\right) !} p^{r_{2}} q^{m-r_{1}-r_{2}} \frac{\left(\lambda_{\mathrm{h}} r_{2}-\lambda_{l} r_{2}-\lambda_{l} r_{1}+\lambda_{l} m\right)}{\left(s+\lambda_{\mathrm{h}} r_{2}-\lambda_{l} r_{2}-\lambda_{l} r_{1}+\lambda_{l} m\right)} \\
& {\left[\frac{d}{d s}(\bar{f}(s))\right]_{s=0}} \\
& =-\left[\sum_{r_{1}=0}^{m} \sum_{r_{2}=0}^{m-r_{1}}(-1)^{m-r_{1}+1} \frac{m !}{r_{1} ! r_{2} !\left(m-r_{1}-r_{2}\right) !} p^{r_{2}} q^{m-r_{1}-r_{2}} \frac{\left(\lambda_{\mathrm{h}} r_{2}-\lambda_{l} r_{2}-\lambda_{l} r_{1}+\lambda_{l} m\right)}{\left(s+\lambda_{\mathrm{h}} r_{2}-\lambda_{l} r_{2}-\lambda_{l} r_{1}+\lambda_{l} m\right)^{2}}\right]_{s=0} \\
& {\left[\frac{d}{d s}(\bar{f}(s))\right]_{s=0}} \\
& =-\sum_{r_{1}=0}^{m} \sum_{r_{2}=0}^{m-r_{1}}(-1)^{m-r_{1}+1} \frac{m !}{r_{1} ! r_{2} !\left(m-r_{1}-r_{2}\right) !} p^{r_{2}} q^{m-r_{1}-r_{2}} \frac{1}{\left(\lambda_{\mathrm{h}} r_{2}-\lambda_{l} r_{2}-\lambda_{l} r_{1}+\lambda_{l} m\right)}
\end{aligned}
$$

Substituting equation (18) in (15) we obtain the mean time to recruitment,

$E(T)=\sum_{i=r}^{n} n C_{i}\left\{\frac{\mu+i \theta}{i \theta}-(n-i) C_{1} \frac{\mu+(i+1) \theta}{(i+1) \theta}+\cdots+(-1)^{n-i} \frac{\mu+n \theta}{n \theta}\right\} \times$ 


$$
\sum_{r_{1}=0}^{m} \sum_{r_{2}=0}^{m-r_{1}}(-1)^{m-r_{1}+1} \frac{m !}{r_{1} ! r_{2} !\left(m-r_{1}-r_{2}\right) !} p^{r_{2}} q^{m-r_{1}-r_{2}} \frac{1}{\left(\lambda_{\mathrm{h}} r_{2}-\lambda_{l} r_{2}-\lambda_{l} r_{1}+\lambda_{l} m\right)}
$$

The mean time for recruitment can be explicitly calculated using (16) and (19) for different possible combinations of the values of $n$ and $r$.

\section{Conclusions}

The influence of the hypothetical parameter on the performance measure can be studied numerically with the help of MATLAB.

\section{References}

[1]. Sridharan.J, Parameswari.K and Srinivasan.A, A stochastic model on time to recruitment in a two grade man power system based on order statistics, International Journal of Mathematical Sciences and Engineering Applications 6(5) (2012):23-30.

[2]. Sridharan.J, Parameswari.K and Srinivasan.A, A stochastic model on time to recruitment in a two grade man power system involving extended exponential threshold based on order statistics, Bessel Journal of Mathematics 3(1) (2013):39-49.

[3]. Sridharan.J, Parameswari.K and Srinivasan.A, A stochastic model on time to recruitment in a two grade man power system involving extended exponential and exponential threshold based on order statistics, Archimedes Journal of Mathematics 3(1) (2013):41-50

[4]. Sridharan.J, Parameswari.K and Srinivasan.A, A stochastic model on time to recruitment in a two grade man power system based on order statistics when the threshold distribution having SCBZ property, Cayley Journal of Mathematics 1(2) (2012): 101-112

[5]. Parameswari.K, Sridharan.J, and Srinivasan.A, Time to recruitment in a two grade manpower system based on order statistics , Antarctica Journal of Mathematics 10(2) (2013):169-181.

[6]. Barthlomew.D.J, and Forbes.A.F, Statitical techniques for man power planning, John Wiley \& Sons, (1979).

[7]. Grinold.R.C, and Marshall.K.J, Man Power Planning, North Holland, Newyork (1977).

[8]. Vidhya.S, A study on some stochastic models for a multi graded manpower system , Ph.D thesis, Bharathidasan University (2011). 